\section{Diabetes' sexy angle}

\section{By Lauren Martz, Staff Writer}

Researchers at Harvard University and the University of California, Los Angeles suggest that plasma levels of the sex hormone regulator SHBG could help predict risk of type 2 diabetes. ${ }^{1}$ In addition, the protein's potential involvement in the development of the disease could make it a therapeutic target.

Determining which individuals are at risk for type 2 diabetes is important because preventive measures such as diet and exercise can help avoid disease development. Risk factors for type 2 diabetes include high body mass index (BMI), large waist circumference and levels of biomarkers such as C-reactive protein (CRP) and hemoglobin Alc (HbA1c).

Levels of both free and bound sex hormones also have been linked to type 2 diabetes. ${ }^{2}$ Sex hormone-binding globulin (SHBG), a protein that sequesters sex hormones and controls their levels in circulation, thus could be a good candidate as a disease marker.

Indeed, low levels of SHBG were shown in 2005 to correlate with poor glycemic control. ${ }^{3}$ Two years later, researchers found that different variants of SHBG affected the levels of circulating globulin and that these levels were associated with insulin resistance. ${ }^{4}$

The missing link, however, was long-term, prospective data correlating SHBG levels with the risk of developing type 2 diabetes. Now, a team led by Simin Liu, professor and director of the Center for Metabolic Disease Prevention and professor of medicine at UCLA, thinks it

\section{"By directly linking SHBG with diabetes risk at both genetic and plasma levels, our data suggest that SHBG may have important biological effects that go beyond simply regulating sex hormones in the blood."}

-Simin Liu, University of California, Los Angeles rs6259 was associated with a $10 \%$ higher level of SHBG, whereas rs6257 was associated with a $10 \%$ lower level, predicting lower and higher risk of disease, respectively. show that, in direct comparison, SHBG seems to outperform not only traditional risk factors but also some newer risk predictors such as

\section{Earlier detection for earlier intervention}

In addition to SHBG's predictive power versus other diabetes risk factors, Liu thinks SHBG protein levels also are predictive of a patient's of developing the disease early.

Liu told SciBX that both genotypic and phenotypic data on SHBG can predict the risk of developing type 2 diabetes in 8-10 years among arently healthy individuals.

Jeffrey Johnson, principal scientist at Metabolex Inc., agreed that testing for levels of SHBG could allow earlier prediction of diabetes risk than testing for indicators such as CRP or HbAlc.

He noted that the authors "are suggesting that SHBG levels could be an independent marker for diabetes risk, whereas HbA1c and C-reactive protein appear to be lagging indicators for diabetes or other components of metabolic syndrome that are already underway. The data in the paper suggest that SHBG levels are in part determined by genotype, and the given genotype is obviously present throughout life and is independent of environmental factors such as diet and exercise that influence other markers."

According to Johnson, "hopefully, this has connected the dots. The group published in The New England Journal of Medicine that low levels of SHBG significantly increase the risk of developing the disease.

High levels of plasma SHBG correlated with lower BMI and risk of hypertension and better lipid profiles and CRP levels in postmenopausal women not using hormone therapy. The study included 359 newly diagnosed type 2 diabetes patients and 359 nondiabetic controls recruited from the Women's Health Study.

The analysis showed that individuals with SHBG in the highest quartile had one-tenth the likelihood of developing the disease compared with patients in the lowest quartile. The findings were replicated in an independent cohort of 170 newly diagnosed type 2 diabetic men and 170 controls from the Men's Health Study II.

Genotype analysis further showed that the SHBG genetic variant would result in counseling about the value of lifestyle changes and how these might be particularly valuable to nondiabetics who are nevertheless more likely to become so in the foreseeable future."

Metabolex's MBX-102, a peroxisome proliferation-activated receptor- $\gamma$ (PPARG; PPAR $\gamma$ ) agonist, is in Phase II testing to treat type 2 diabetes. The compound is partnered with Johnson \& Johnson.

"It is my hope that SHBG testing, both for genotypes and plasma phenotype, would be incorporated into routine blood screening for type 2 diabetes. Genotyping and immunoassays should be fairly straightforward and shouldn't be that costly," said Liu.

"Some of the diagnostic companies are aggregating groups of biomarkers to increase the predictive power of their tests. I am sure that all of these groups will consider adding SHBG to their list of markers," 


\section{TARGETS \& MECHANISMS}

said Johnson. "Over time, this should also allow the diabetes prediction community to assess the relative prognostic power of a newer marker like SHBG."

Liu agreed. "SHBG could be independently predictive of diabetes risk, but we recommend it to be used in conjunction with other wellestablished traditional risk factors," he said.

Altan Onat, emeritus professor of medicine at Istanbul University's Cerrahpasa Medical Faculty, who has worked on the role of SHBG in triglyceride dyslipidemias, thinks the protein is not ready to become part of the standard battery of tests for diabetes risk.

"Though SHBG screening may, indeed, add information additive to waist circumference and to certain blood tests in men and women past 50 years, it is still premature to use SHBG screening in a standard fashion," he said.

For example, Onat said, a concentration value for SHBG needs to be established under which patients are at a high risk for developing type 2 diabetes.

Johnson said the NEJM findings need to be validated with a larger number of subjects and added that "the next steps are probably learning more about the physiological differences between individuals with low and high levels of SHBG."

Liu agreed that larger studies are in order. He also said his group needs to run studies in "more ethnically diverse populations so we can establish potential race-specific population reference values for SHBG and sex hormones in relation to risk of diabetes."

\section{Therapeutic target}

Because low SHBG levels correlate with an increased risk of diabetes, increasing levels of the protein could be a point of therapeutic intervention for at-risk patients.

"The most exciting part of this story is the potential for a role for low SHBG levels in the etiology of type 2 diabetes, in which case you have both a strong predictive marker and new ways of thinking about treating diabetes and perhaps preventing it," said Johnson. "It is possible to envision a class of therapeutics that could be used to slow the progression to hyperglycemia in people at high risk.

He did caution that therapeutics that displace SHBG-binding sex hormones could have a host of unintended side effects.

Nevertheless, Johnson told SciBX that the results in the NEJM article may lead drug discovery groups to carefully consider the therapeutic potential of SHBG.

"Our data should have implications for developing treatment and preventive measures that specifically aim at raising SHBG levels," said Liu.

UCLA has filed a patent application covering the work. Liu said the university is in exclusive licensing negotiations with an unnamed company but noted that other licensing or partnering opportunities could still be available.

Martz, L. SciBX 2(35); doi:10.1038/scibx.2009.1331

Published online Sept. 10, 2009

\section{REFERENCES}

1. Ding, E. et al. N. Engl. J. Med.; published online Aug. 5, 2009; doi:10.1056/NEJMoa0804381

Contact: Simin Liu, University of California, Los Angeles, Calif. e-mail: siminliu@ucla.edu

2. Ding, E. et al. Diabetologia 50, 2076-2084 (2007)

3. Sutton-Tyrrell, K. et al. Circulation 111, 1242-1249 (2005)

4. Berndt, S. et al. Cancer Epidemiol. Biomarkers Prev.16, 165-168 (2007)

COMPANIES AND INSTITUTIONS MENTIONED

Harvard University, Boston, Mass.

Istanbul University, Istanbul, Turkey

Johnson \& Johnson (NYSE:JNJ), New Brunswick, N.J.

Metabolex Inc., Hayward, Calif.

University of California, Los Angeles, Calif. 